\title{
APPLICATON OF PROCESS MODELING AND RAPID PROTOTYPE PATTERNS IN THE DEVELOPMENT OF THIN WALL IN718 INVESTMENT CASTINGS
}

Clay Carlson, Gerald Schurkamp, Howmet Corporation Dan Wei, General Electric Aircraft Engines

Process modeling and rapid prototyping are being utilized to transition IN718 components that were traditionally manufactured as fabrications, to investment castings. The thin walls (0.040 inches) and geometry of the components are difficult to cast, but with the aid of process modeling simulations, gating schemes and process parameters can be examined and defined prior to committing to expensive casting trials. Two principle objectives of the modeling efforts are to predict mold fill and metallurgical defects, such as porosity. Successful simulations are verified through foundry casting trials utilizing rapid prototype patterns made with the SLA process, thus eliminating the need for hard tooling. The combination of these two technologies reduces development time, risk and cost, and opens up the potential for a new product line of investment castings. 


\section{Introduction}

The investment casting process has improved over time to allow component designers greater flexibility in geometric detail as well as dimensional control. These improvements can be traced to advancements in $\mathrm{CAD} / \mathrm{CAM}$ utilization, investment casting material development, and process control. However, it is difficult to transition existing components traditionally manufactured with alternative methods to investment castings. The underlying reasons are : 1) there are large up-front costs in creating the tooling required to generate wax patterns, and 2) successful castings, especially for new parts, are the result of multiple foundry trials which allow the casting engineer to optimize the casting process. The Materials Affordability Initiative program (under the auspices of the United States Air Force) is designed to develop the required casting technologies to allow parts that were traditionally manufactured as fabrications to be transitioned to more cost effective investment castings.

Traditionally, the discriminator between fabrications and investment castings has been thin part cross sections. Fabrications often use formed sheet stock for thin cross sections. However, sheet forming is restrictive in creating finely detailed part features, and the ensuing welded elements of the fabricated part are susceptible to distortion during the welding process. Castings are quite capable of providing fine part detail, but are susceptible to defects in thin sections during the metal pouring and solidification process.

The approach of the MAI Thinwall Program is to develop new casting techniques targeted specifically at thin-wall castings. The improvements include investment shell system materials and processing, metal delivery geometry (gating), as well as casting process controls (alloy pouring temperature, mold preheat, etc). To allow flexibility and reduce costs, the patterns for these trials were made with the QuickCast SLA process. Prior to the foundry trials, process modeling simulations were performed to predict the results of the selected shell system, gating configuration and casting process parameters.

The component under study for this report is an aircraft engine accelerator nozzle. The component as shown in Figure 1, is composed of small stationary, low aspect ratio airfoils confined between inner and outer shrouds. The accelerator nozzle provides no engine structural support, and the major design issues are geometric accuracy (of the accelerator airfoils), and total component weight. Therefore, as a cast structure, the challenges are to maintain the geometric accuracy inherent in a casting with walls as thin as possible.

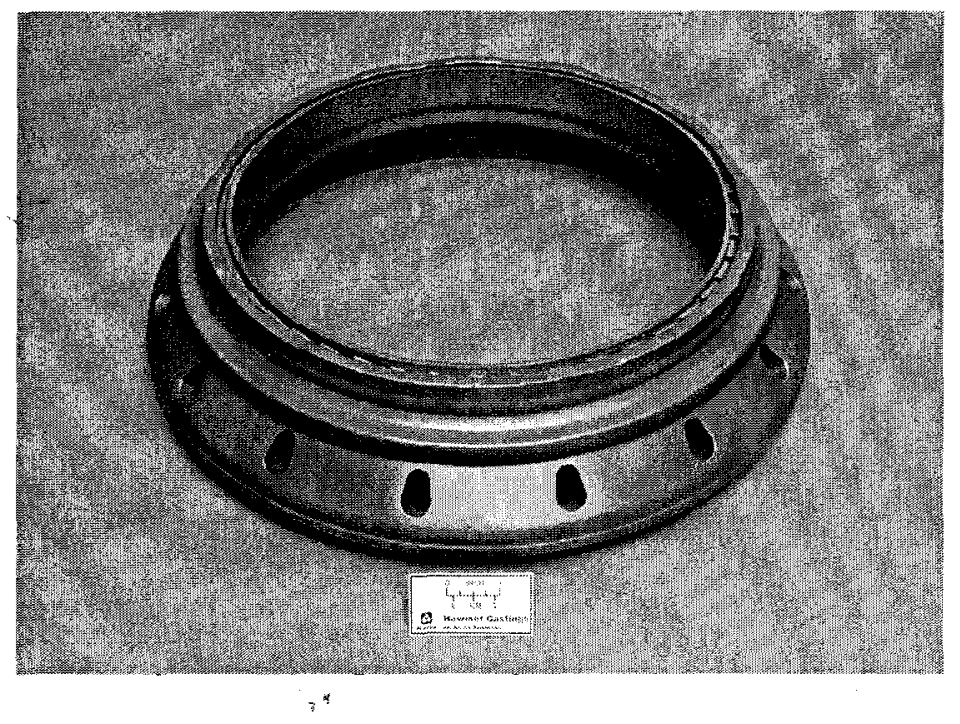

Figure 1: General Electric Aircraft Engine Accelerator Nozzle

\section{Rapid Pattern Production}

The rapid prototype patterns are produced using a sterolithography apparatus (SLA). The QuickCast algorithm for producing rapid patterns was employed to generate an honeycomb structure, thus minimizing the stress on the shell during burnout. The patterns were built within Howmet's own Rapid Prototyping department, thus providing direct control of the shrink factors to insure adherence to the 
0.045 inch wall thickness. The patterns were all inspected with a Coordinate Measuring Machine (CMM) to verify critical feature dimensions. The QuickCast patterns were assembled into molds in the same manner as traditional wax patterns. Wax gating and runner systems are connected to the rapid pattern to form the mold assembly. The assembled molds are then invested with a series of dips in ceramic slurries and stucco. The wax and resin patterns are removed from the mold with a thermal process and the mold is prepared for metal pouring.

The two large risk items of a casting development program are the pattern tooling costs and the required foundry trials to develop a casting process. The use of rapid patterns addresses this first risk item by drastically reducing the cost of the patterns. Hard tooling costs for this type of component is approximately $\$ 280,000$; with 14 weeks of tool build cycle. The QuickCast patterns were approximately $\$ 3400$. The large number of small aspect ratio airfoils in the component requires a substantial amount of finishing, and hence, cost to prepare the pattern for the investment process. However, these same features drive up the hard tooling costs and impede the conventional wax pattern production rates. The second risk element: casting process development is addressed with process modeling.

\section{Process Modeling}

Process modeling is the computer simulation of the casting process. This simulation is performed in the context of a finite element model (FEM), and includes all the geometry of the actual foundry mold including pour cups, runner systems, investment shell and insulation. The electronic data that is used to generate the QuickCast patterns also is the genesis of the FEM model. The use of product symmetry is often employed in modeling to improve the computational performance. Figure 2 depicts the CAD definition of one fourth of the part.

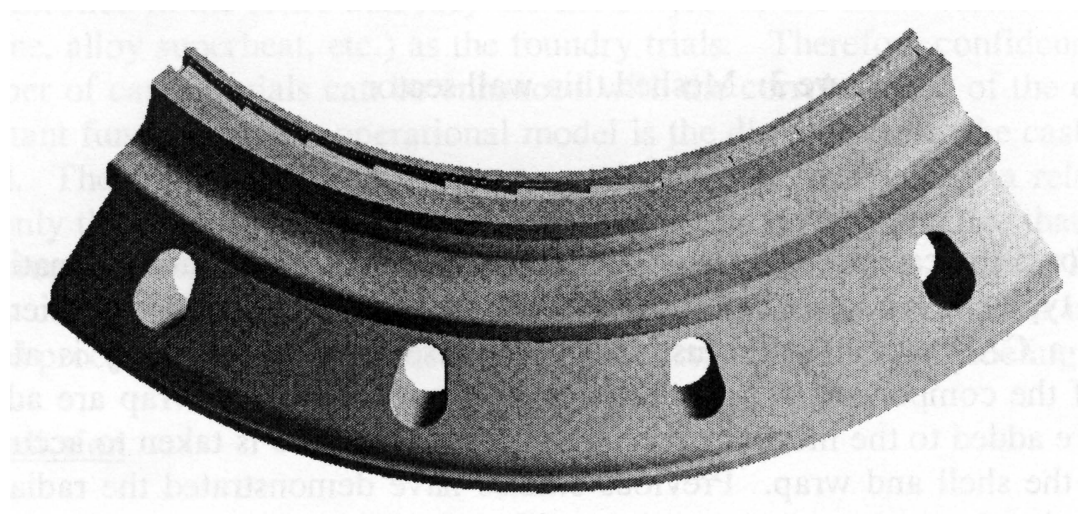

Meshing

Figure 2: Computer Aided Design Model (Quarter symmetry)

The thin wall nature of this part does have implications upon the meshing strategy. The analysis code solves the momentum and energy equation in a coupled fashion. The fluid flow analysis has the familiar no-slip condition at the wall, therefore, the solution is highly influenced by the nature and quality of the FEM grid in the thin wall sections of the part. As in most circumstances, the optimum grid is a balance of being fine enough to capture the physical phenomena without incurring any numerical penalties. From a practical standpoint, the modeling effort supported the casting trials and, therefore, had to be timely enough to provide gating/runner and process information. Preliminary numerical studies of idealized test geometry were used to probe the mesh quality issues. Results from this investigation indicated that the element count had to be larger than for conventional analyses with larger wall thickness. Attempting to create faster solving meshes with fewer elements resulted in the prediction of non-fill for the idealized geometry that was not corroborated by casting trials. For this part, the 
triangulated surface edge length needed to be on the same order as the wall thickness $(0.045$ inches). The solid mesh, which emanates from the surface mesh, had a specified aspect ratio of 1.0. Although, the resulting solid mesh had a distribution of aspect ratio, the mesh in the critical areas appeared to be of good quality. Casting trials (which will be discussed in more detail) corroborated the simulated fill patterns. Figure 3 illustrates the mesh of the part without any of the attached gating/runner systems.

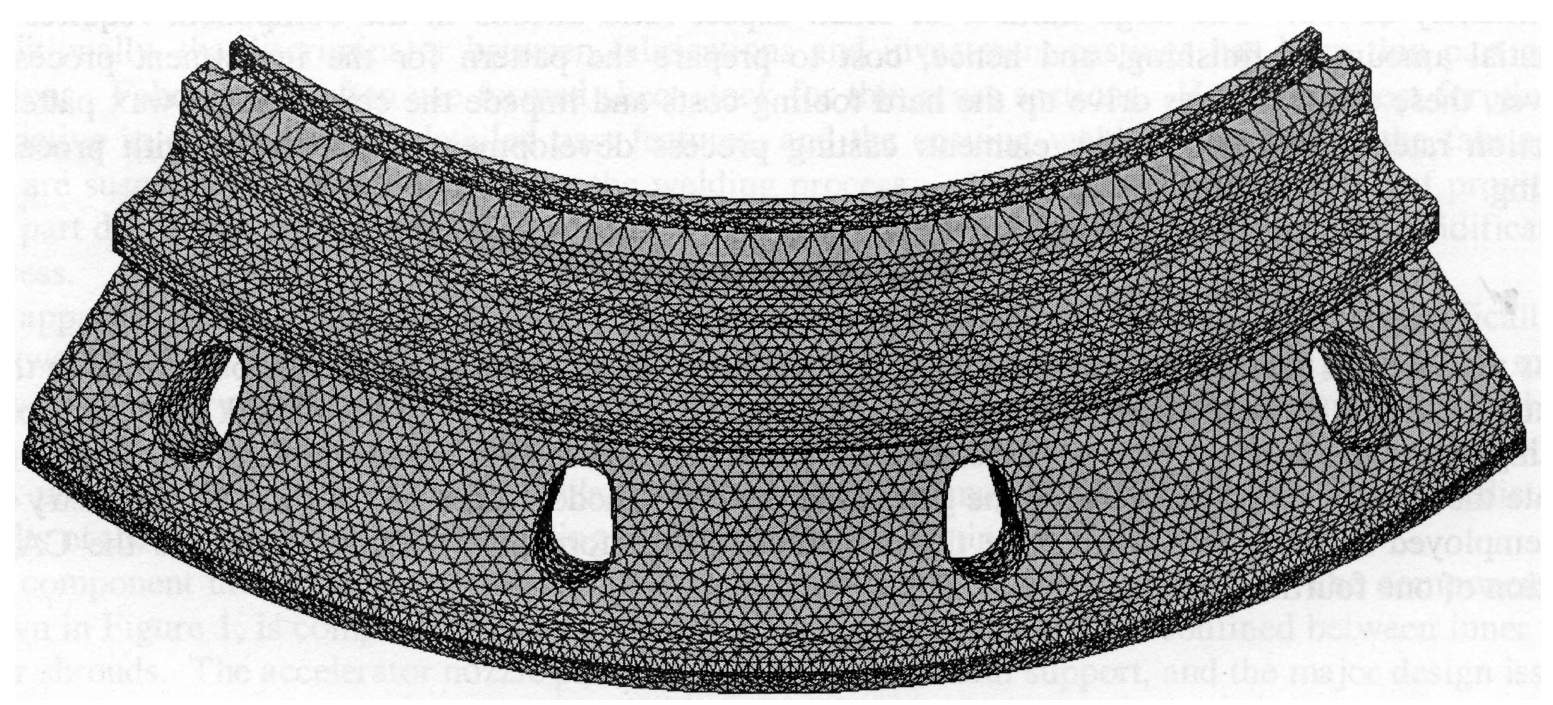

Figure 3: Meshed thin wall sector

\section{Gating}

Process modeling affords the casting engineer the ability to efficiently evaluate alternative gating. The first gating scheme is typically a conjecture by the engineer based upon previous experience. The gating scheme is created in a CAD system with customer part present. This geometry is also meshed and joined to the mesh of the component. The investment shell and insulation wrap are additional sets of finite elements that are added to the model. In each of these cases, care is taken to accurately represent the true geometry of the shell and wrap. Previous studies have demonstrated the radiation dominated heat transfer, which exists in a vacuum casting, is significantly affected by the modeled geometry. The resultant radiation view factors affect the local cooling rate of the casting and hence, the formation of metallurgical defects. Additional gating configurations are derived from the process modeling results and the casting trials.

\section{Process Parameters}

The casting process parameters are as important as accurate geometry and meshing. These details include the furnace environment, mold preheat conditions, alloy pouring temperature and pour time. The SCP (susceptor cast process) is a Howmet proprietary process derived specifically for thin wall castings. The process application and details have been developed with the use of process modeling, and continue to be refined as the MAI program progresses. Given the large number of combinations of gating/runner geometry and process parameters, process modeling provides a logical and efficient approach to developing a successful casting process. Beyond the quantitative results provided by the method, process modeling illustrates to the casting engineer details of the process that are impossible to discern from casting trials. Fluid flow patterns and local mold cooling rates can be graphically portrayed and compared with alternate gating and process parameters. 
The analytical models were solved on a Silicon Graphics Origin 2000 computer with R10000 $250 \mathrm{MHz}$ chips and $6 \mathrm{~GB}$ of ram storage. The finite element software use to predict solidification was ProCAST, developed and marketed by UES. ProCAST is developed specifically for the casting industry, and has many features tailored towards foundry processes. The code is capable of determining the fluid free surface for gravity or momentum dominated flows. Since these models have a relatively short pour time, the free surface was calculated as a momentum dominated flow.

The different gating schemes resulted in multiple models with various element counts, ranging from 700,000 to $1,000,000$ tetrahedral elements. Solution time was between 44 and 50 hours. This time, when added to the original model preparation time of approximately 1 week, implies that an analytical casting solution is feasible within a two-week time frame. Of course, geometric modifications beyond the original configuration don't take as long as previous CAD and FEM meshing can be recycled into the new configurations. Changes in process variables can be turned around in a matter of hours. These time scales are amenable to a development program and allows the process modeling effort to be the predictor of the actual foundry trial. Ideally, multiple models can be executed and examined prior to the next foundry trial.

\section{Modeling Results}

The MAI Thinwall program has been structured to utilize process modeling combined with foundry trials, and involves multiple parts with materials other than IN718. In each case, the process model prediction is performed prior to the casting trial. To date, the ratio of process simulations-to-casting trials is better than $2: 1$. That is, there are two simulations performed for each casting trial. This model of operation, performing casting simulations coupled with actual foundry trials, provides two important functions. First, the simulations augment the knowledge that is gained in the casting trial process. The simulations are controlled in the sense that they are not subject to the same variability of process (shell thickness, pour time, alloy superheat, etc.) as the foundry trials. Therefore confidence in a statistically insignificant number of casting trials can be enhanced with the corroboration of the casting simulation. The second important function of this operational model is the direct inverse: the casting trial augments the process model. There is a plethora of program variables and empirical data related to the casting simulation. It is only through the continuous feed back of actual casting practice that process modeling becomes an effective predictive tool. In a development program of a new product line involving new processes, foundry casting trial feed back is critical. The next sequence of figures is demonstrative of the aforementioned process. It is representative of the interaction of process modeling and casting trials.

\section{Macroporosity Prediction}

ProCAST software contains an embedded macroporosity model that tracks isolated islands of hot metal during the cooling process. Porosity occurs when these pockets undergo density changes and there is no path for flowing metal to replace the created voids. Figure 4 illustrates the results of a particular gating, insulation configuration and set of process parameters. The clear portions of the picture indicate voidfree areas, and the shaded portions indicate areas that are likely to have voids. For this case, there is a large area of porosity around the outer flange near the turning airfoils and includes some of the airfoils themselves. There are also small pockets of void underneath the elongated holes that connect the outer and inner flanges. Subsequent $x$-rays of the casting partially corroborated these results, but with some caveats. The $x$-rays confirmed the porosity in the regions below the elongated holes but the model overestimated the shrinkage in the upper portion of the casting. Post cast examination of the mold gating compared to the modeled gating indicated some geometrical differences that could be attributed to the observed model-cast trial results. 


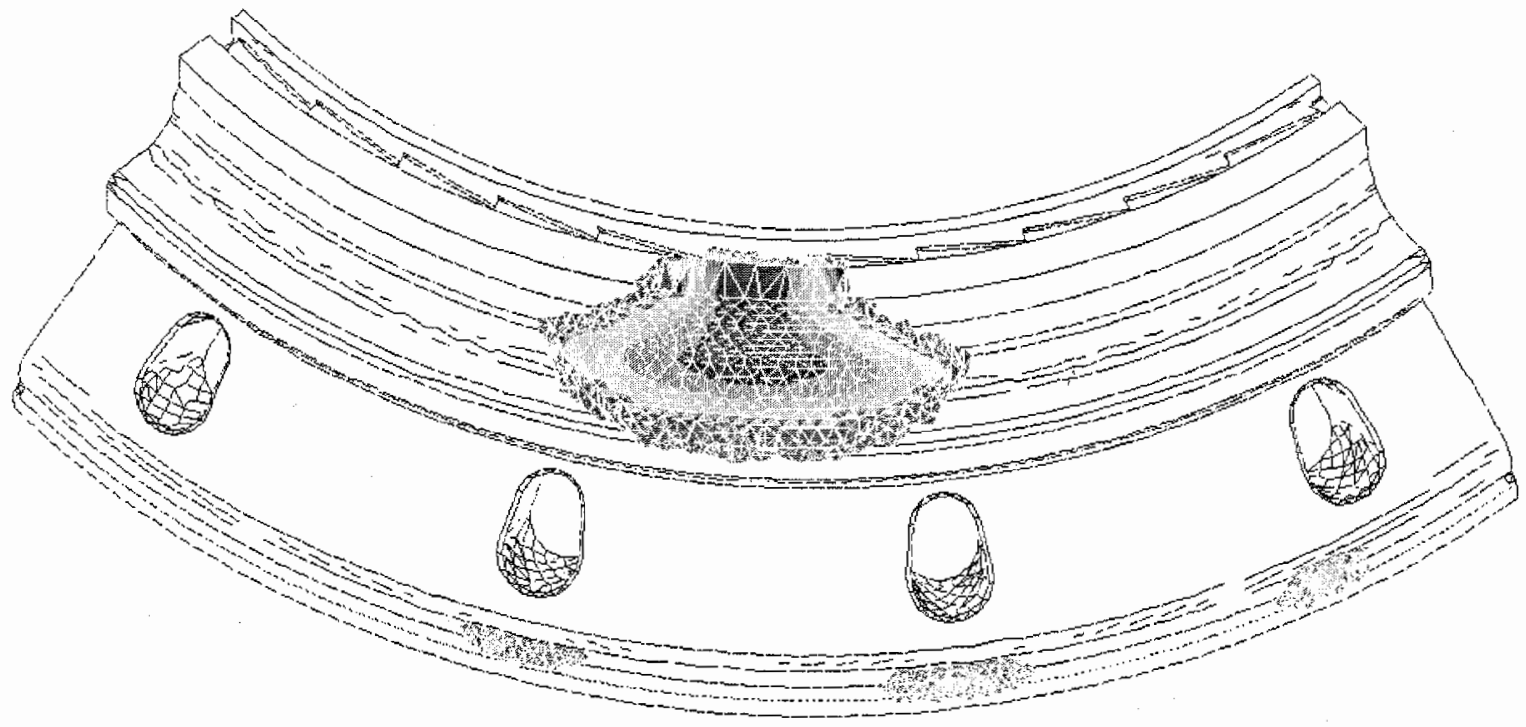

Figure 4. Macroporosity of gating configuration 1

However, to minimize this sensitivity, a second gating scheme was developed. The results, illustrated in Figure 5, at first glance, appear to be decidedly worse than those of the previous configuration. However, it must be noted that this simulation was void of any insulation wrap (which for production would be the desired mold setup). However, the gating change did eliminate the smaller pockets of porosity under the elongated holes. Based upon this simulation, it was decided not to submit this configuration for a foundry casting trial.

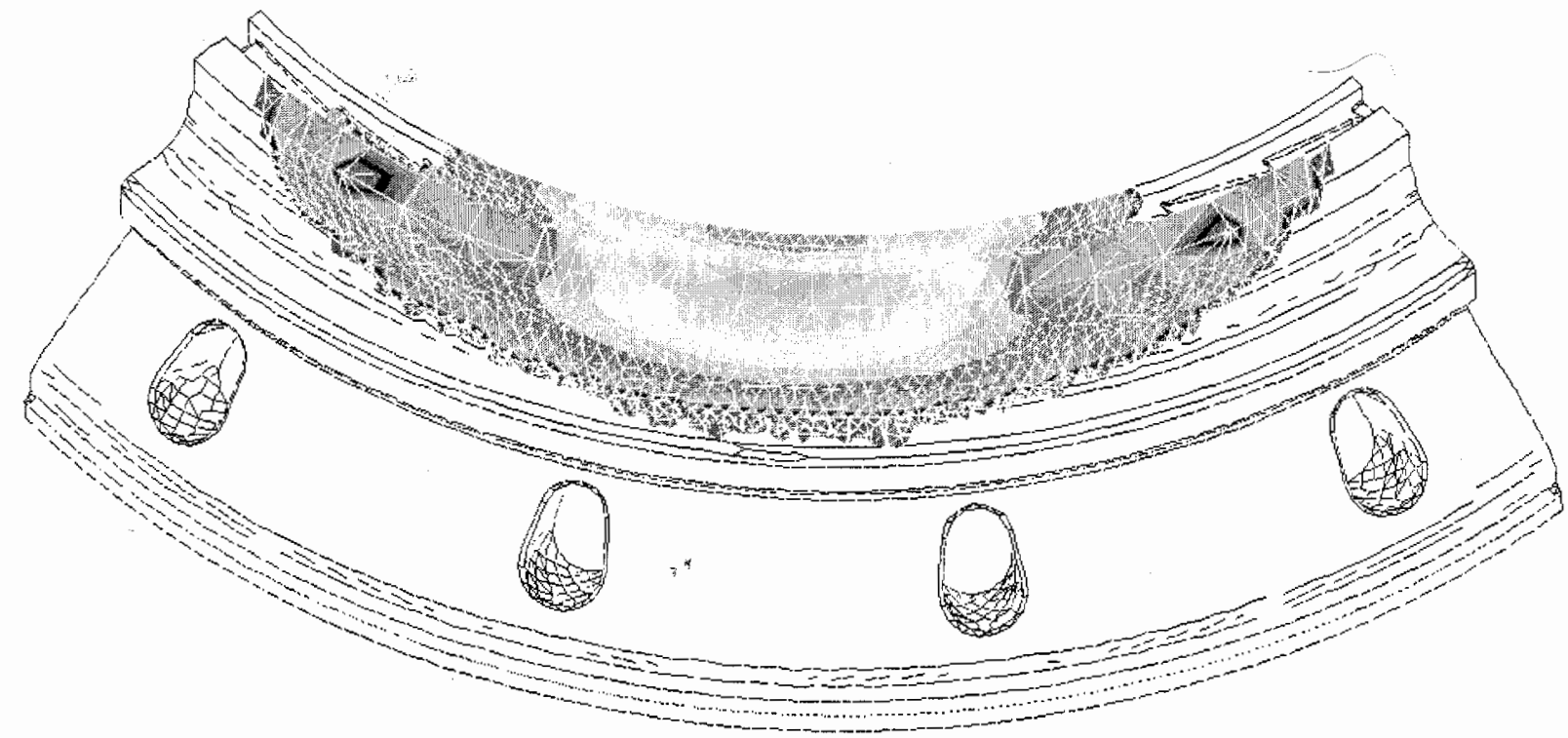

Figure 5: Macroporosity for gating configuration 2

A third simulation, using the gating system of Figure 5, but with the addition of an insulation wrap in the vicinity of the upper gating was performed with the results depicted in Figure 6 . This configuration 
shows a largely void-free solution (there is a small amount on the outer flange). This result was confirmed by the casting trial.

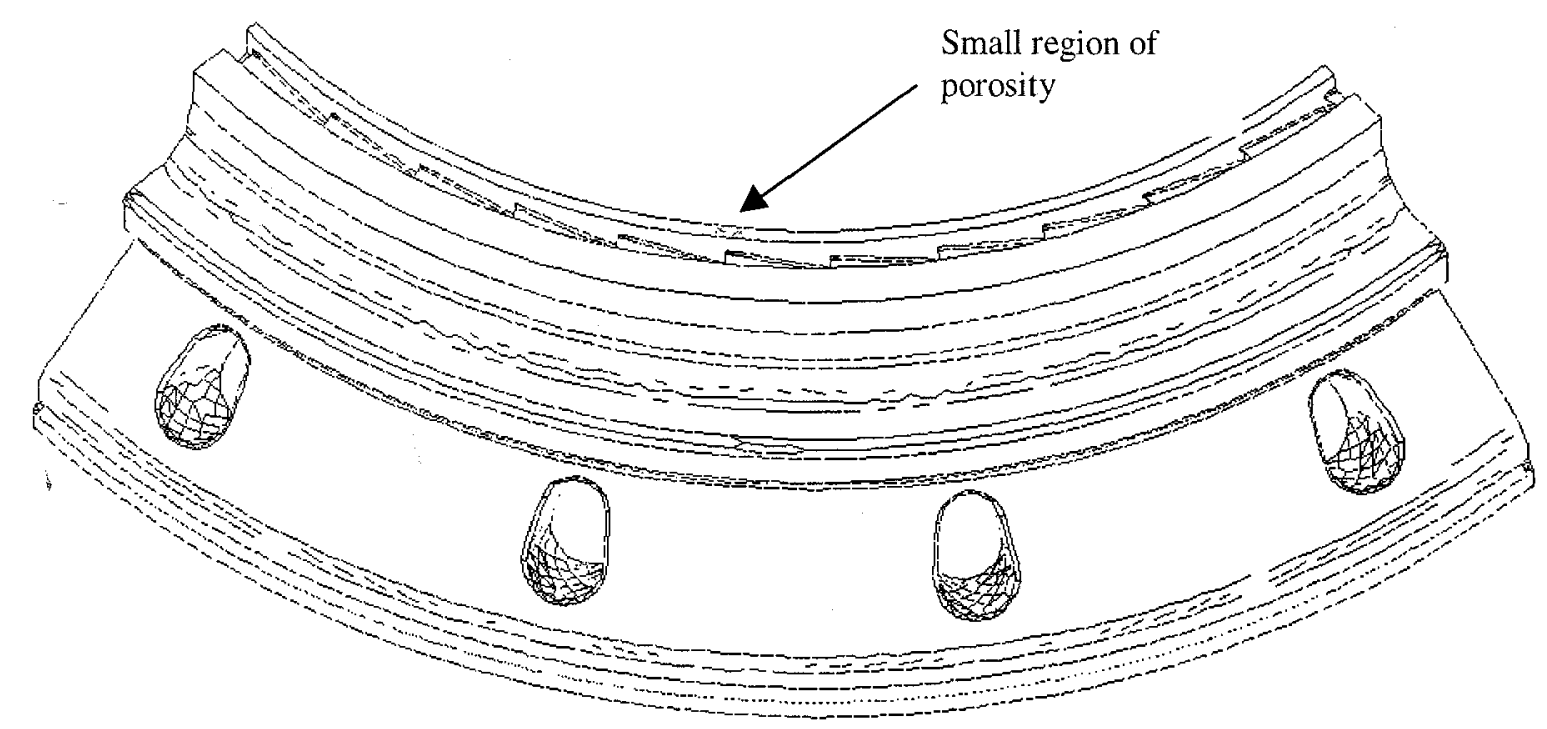

Figure 6: Macroporosity for gating configuration 2 with insulation wrap

\section{Summary}

The development of casting processes for new product categories is difficult due to the large initial tooling costs and the casting process development time. Using conventional foundry practice, the risk and lead times often thwart the development of new casting techniques that could yield new categories of cast products. The use of rapid prototype patterns and process modeling address both these issues and allow for a cost and time efficient means of developing new technologies.

\section{$\underline{\text { References }}$}

1. R.E. Taylor, "Thermophysical Properties of IN 718", Thermophysical Properties Research Laboratory, (Report TPRL-1347-IN718, Purdue University, 1994).

2. Eric Foos, Boyd Mueller, "Casting Conversions Utilizing Computer Aided Engineering", (Presented at Investment Casting Institute, 2000).

3. Richard K Foran, Ty Hansen, Boyd Mueller, "Recent Advances in the Application of Modeling to the Investment Casting Process", (Presented at AIM conference, 1995). 